\title{
Zoom.us y sus potencialidades para generar aprendizajes significativos en odontología restauradora directa e indirecta: Experiencia inédita.
}

Zoom.us and its potential to generate meaningful learning in direct and indirect restorative dentistry: Unprecedented experience.

Miguel Ángel Saravia-Rojas 1,a, Mary Fukuhara-Nakama ${ }^{1, b}$

RESUMEN

La COVID-19 generó una emergencia sanitaria que impidió la presencialidad a las aulas en la educación superior afectando el desarrollo académico de la enseñanza de la odontología y de todas las ciencias. Esto ocasionó que las instituciones académicas se organicen de manera adecuada para responder rápida y oportunamente para poder asumir estos nuevos retos. El uso de plataformas para video conferencias que permitan reemplazar las habituales clases magistrales fue la respuesta que se obtuvo y su implementación se realizó en el más breve plazo. El resultado de esta implementación obligó a las instituciones académicas a decidir por algunas de ellas para responder eficazmente al nuevo reto. En este documento compartimos nuestra experiencia con la plataforma Zoom.us durante los cursos de Clínica Integral del Adulto CIA I- 2021 y Clínica Integral del Adulto CIA III -2021 brindado a los alumnos del V y VII semestre respectivamente de la carrera de Estomatología. En esta experiencia se describe la percepción del uso de las herramientas que dicha plataforma posee y cómo ellas ayudan a generar experiencias de aprendizaje significativas.

PALABRAS CLAVE: Zoom. us, educación dental, aprendizaje dental, simulación dental, Odontología Restauradora.

Facultad de Estomatología, Universidad Peruana Cayetano Heredia. Lima, Perú.

Profesor Principal, Cirujano Dentista, Maestría en Estomatología, Doctor en Estomatología

Profesor Asociado, Cirujano Dentista, Maestría en Estomatología, Maestría en Educación con mención en Docencia e Investigación en Educación Superior. 


\begin{abstract}
COVID-19 generated a health emergency that prevented classroom attendance in higher education, affecting the academic development of the teaching of dentistry and all sciences. This gave rise to academic organizations to organize themselves adequately to respond quickly and in a timely manner to be able to take on these new challenges. The use of video conference platforms to replace the usual master classes was the response that was obtained and its implementation was carried out in the shortest time. The result of this implementation forced academic institutions to decide for some of them to respond effectively to the new challenge. In the experience we share our experience with the Zoom.us platform during the CIA I Adult Comprehensive Clinic and CIA III Adult Comprehensive Clinic courses given to the students of the 5th and 7th semesters of the Stomatology career in our house of studies. In this experience, the perception of the use of the tools that said platform has and how they help to generate meaningful learning experiences is shared.
\end{abstract}

KEY WORDS: Zoom.us, dental education, dental learning, Restaurative dentistry, dental Simulation

\section{INTRODUCCIÓN}

La Emergencia sanitaria debido al COVID-19 obliga a que las actividades académicas presenciales pasen a ser de manera remota usando diferentes plataformas como es el caso de Zoom.us, Anymeeting, Skype, Webex, GoogleMeet, GotoWebinar entre otras para poder transmitir las clases magistrales de las instituciones académicas para seguir operando y no detenerse frente a esta eventualidad.

La literatura menciona que las plataformas para videos conferencias requieren de ciertas cualidades $(1,2)$ :

- Gestión amigable para el usuario docentediscente.

- Autonomía para agendar, modificar y/o cancelar clases.

- Capacidad de compartir presentaciones PowerPoint en modo presentador.

- Autonomía para decidir qué grabar y cómo hacerlo.

- Posibilidad de trabajar con grupos de estudiantes y simular desplazamientos.

- Posibilidad de compartir el control de la sesión por coanfitrión.

- Espacio para gestionar preguntas/respuestas.

- Fácil integración con otros recursos $(1,2)$.

Asimismo, Vera en el 2021 (1) manifiesta que estas plataformas deberían contener algunas dimensiones con sus respectivas descripciones que a continuación detallamos:
Amistocidad: Interfaz de plataforma que es fácil de comprender, aprender y utilizar. Se trata de interfaz simple, directa que entrega un acceso rápido a las funciones o comandos de un programa o dispositivo.

Metodologías: Ductilidad de la plataforma para integrar metodologías activas y sistemas de evaluación alternativa o auténtica.

Comunicación: propiedad que permite interactuar de manera directa con los demás, con un enfoque de cara a cara (uno a uno o uno a varios).

Integración: Propiedad que permite comenzar una reunión de inmediato ya sea agendándola o enviando una invitación automáticamente a través de correo electrónico.

Seguridad: Capacidad de un sistema de proteger una reunión mediante diversas medidas, tales como la encriptación, presencia de anfitrión, sala de espera, contraseña, bloqueo, entre otras.

Gestión de soluciones: Capacidad para desarrollar soluciones, con un enfoque participativo como una manera de llegar a la mejor alternativa (1).

\section{Características de la plataforma Zoom.us (3)}

El programa Zoom permite interacciones sincrónicas entre educadores y estudiantes. En este entorno en línea, las personas usan una cámara web $\mathrm{y}$ un micrófono para chatear en tiempo real, lo que 
permite interacciones similares a las que ocurren en el entorno tradicional del aula (4). Hasta 200 personas pueden participar activamente en sesiones en vivo, y 3000 asistentes adicionales pueden ver pasivamente la sesión(5). Aunque este programa se basa en suscripciones con planes educativos a partir de $\$ 1800$ anuales para 20 hosts, está disponible una versión gratuita que limita las sesiones de video a 40 minutos. Debido a la pandemia de COVID-19, la compañía eliminó este límite de tiempo para las cuentas básicas gratuitas para escuelas primarias y secundarias(6).

Los alumnos pueden participar en una variedad de actividades educativas diferentes dentro del entorno de Zoom. Por ejemplo, las actividades relacionadas con la comunicación incluyen saludar a los demás, conferencias en el aula, preguntas y respuestas y discusiones grupales en salas de grupos pequeños (4). Las actividades relacionadas con los materiales comprenden compartir diapositivas o pantallas con estudiantes o educadores, descargar tareas y cargar respuestas a preguntas(4). Las actividades relacionadas con el estudio pueden tomar la forma de contestar preguntas de encuestas, presentar lecciones usando diapositivas o la pizarra, práctica en el aula usando la pizarra o la caja de chat y trabajo en grupo en salas para grupos pequeños(4). Los estudiantes y educadores también pueden reunirse individualmente para discutir el trabajo del estudiante, y estas sesiones se pueden grabar para verlas más tarde (7) al igual que con otros programas en línea sincrónicos, los estudiantes pueden distraerse o participar en tareas múltiples y las clases y conferencias pueden extenderse más de lo previsto. Los estudiantes que utilizan Zoom también pueden experimentar dificultades como conexiones a Internet poco fiables, falta de un entorno silencioso y altavoces o micrófonos inadecuados $(8,9)$.

Al revisar la literatura hemos podido encontrar que las experiencias haciendo uso del Zoom son descritas como favorables y altamente recomendables. Algunos de ellos destacan las funciones que el Zoom presenta para poder facilitar la transmisión de la información, como es el caso de poder grabar la actividad para que las personas puedan realizar de manera asíncrona su revisión. Una ventaja clave de Zoom es su capacidad para grabar y almacenar sesiones de forma segura sin recurrir a software de terceros.
Algunos estudios manifiestan su preferencia de las conferencias en video pregrabadas a las conferencias Zoom en vivo debido a su flexibilidad, conveniencia y efectividad educativa.

Esta característica es particularmente importante en la investigación donde se requiere la protección de datos altamente sensibles. Los estudios describieron comúnmente la tecnología Zoom en términos positivos debido a su conveniencia, facilidad de uso, seguridad, interactividad, características únicas (por ejemplo, compartir pantalla, opción de grabación de video, entre otros) y su capacidad para facilitar conexiones personales entre usuarios. Estos resultados sugieren que Zoom puede servir como una plataforma muy adecuada para recopilar datos de entrevistas cualitativas en comparación con otras tecnologías $(10,11,12)$.

\section{Herramientas de Zoom y sus posibilidades de generar aprendizajes significativos}

El Zoom presenta varias herramientas que permiten una comunicación efectiva para con los alumnos. Destacamos una de las que hemos podido usar que nos ha llamado poderosamente la atención para poder sacarle mejor provecho a las actividades realizadas en la maqueta-UPCH. Las imágenes que mostramos a continuación ponen en evidencia la función anotar. Herramienta versátil que acepta usar diferentes íconos que permiten explicar con detalle las instrucciones que brinda el docente y que además proporcionan oportunidades para señalar los avances y eventuales retrocesos que la experiencia vía remota puede poner en evidencia y brindar retroalimentación efectiva para generar los avances correspondientes así como las correcciones con el mismo propósito. En la experiencia se comparten algunas de las actividades realizadas en los cursos de la Clínica Integral del Adulto I-2021 y Clínica Integral del Adulto III-2021 (Figuras 1, 2, 3 y 4).

\section{Resultados de la encuesta}

Para conocer la opinión de los que participaron de esta experiencia se aplicó una encuesta a los docentes y a los estudiantes, se obtuvo la respuesta de 14 docentes y 49 estudiantes. Los reactivos fueron validados por dos docentes del área. La participacion de los docentes y alumnos fue voluntaria. 

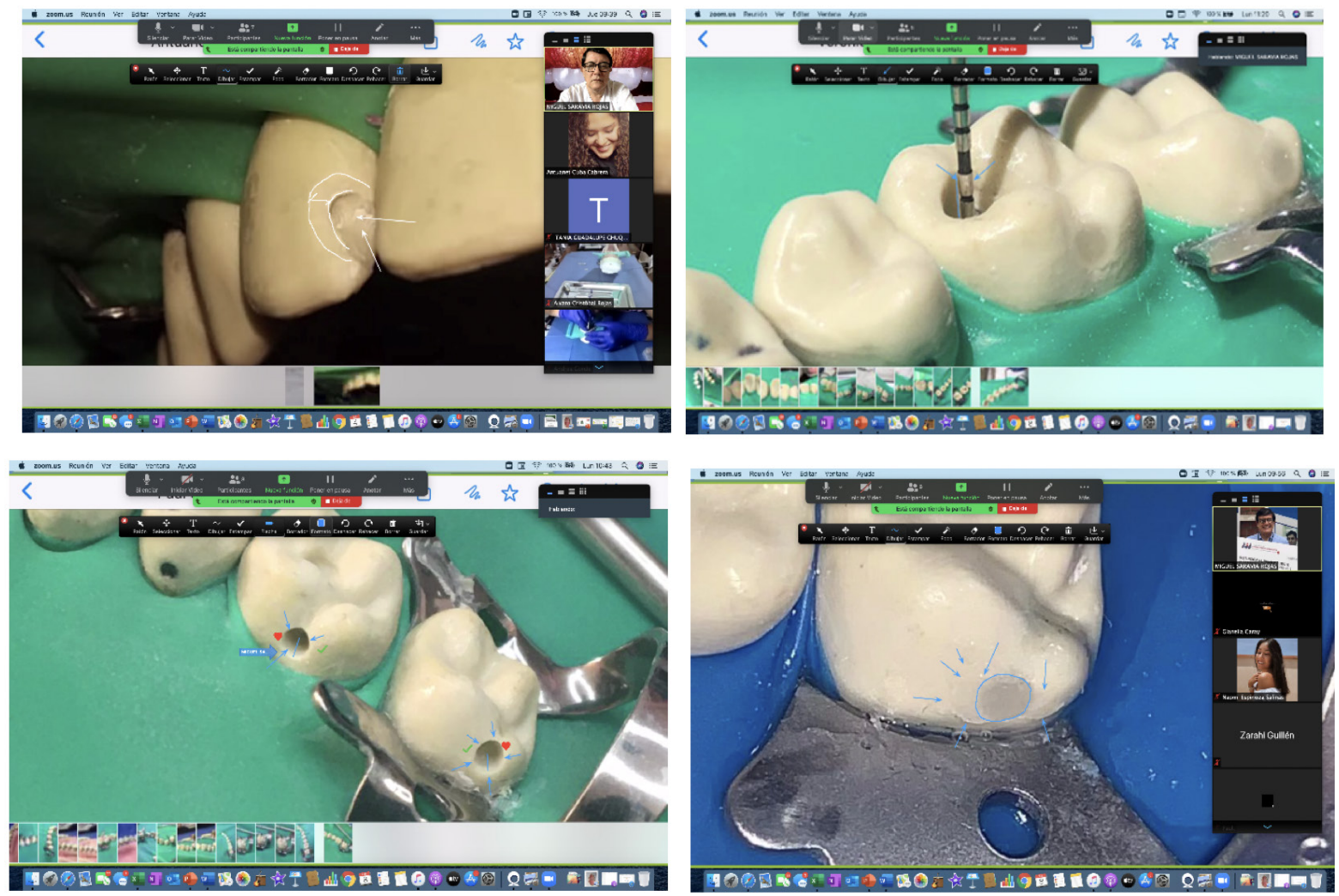

Figura 1. Uso de la herramienta anotar para señalar características importantes a tener en cuenta en una práctica remota de restauraciones en maqueta.
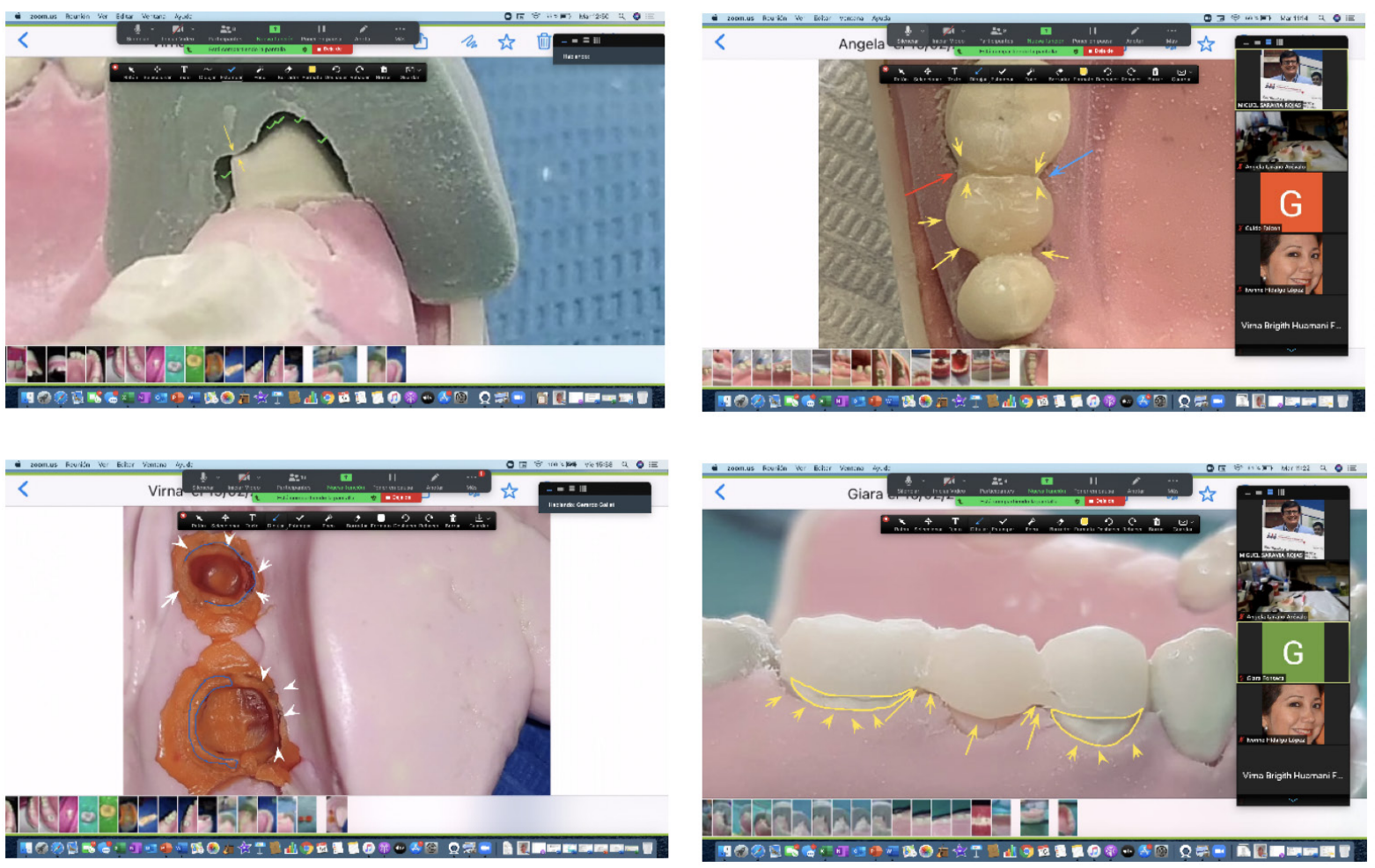

Figura 2. Uso de la herramienta anotar para señalar características importantes a tener en cuenta en una práctica remota de prótesis en maqueta. 

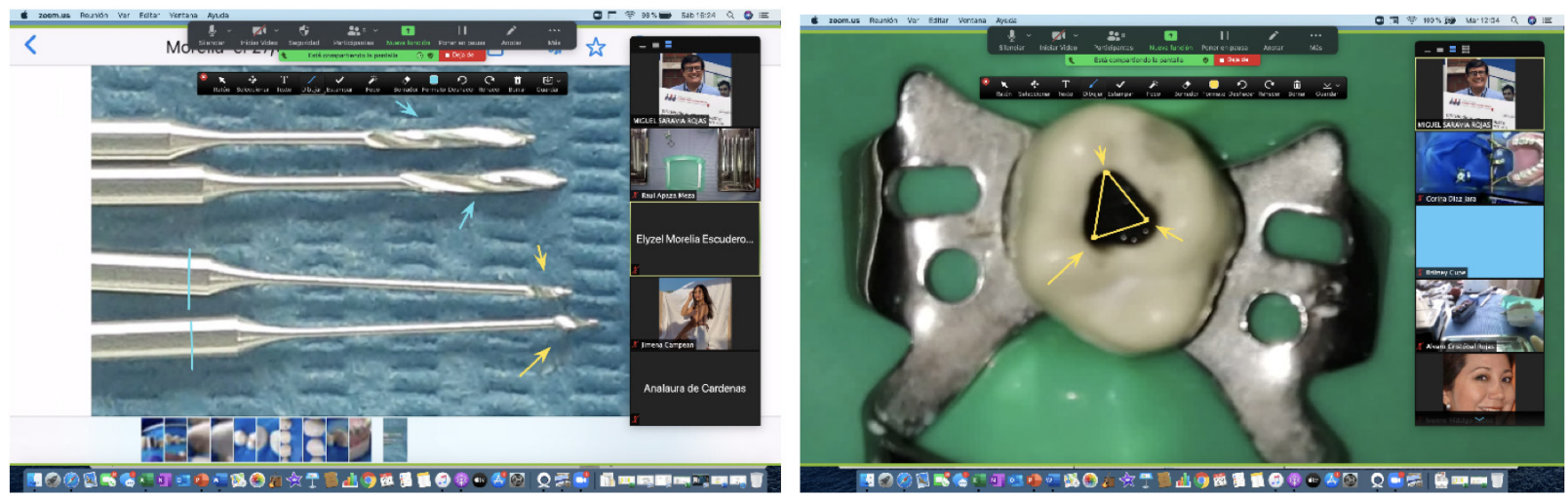

Figura 3. Uso de la herramienta anotar para señalar características importantes a tener en cuenta en una práctica remota de endodoncia en maqueta.
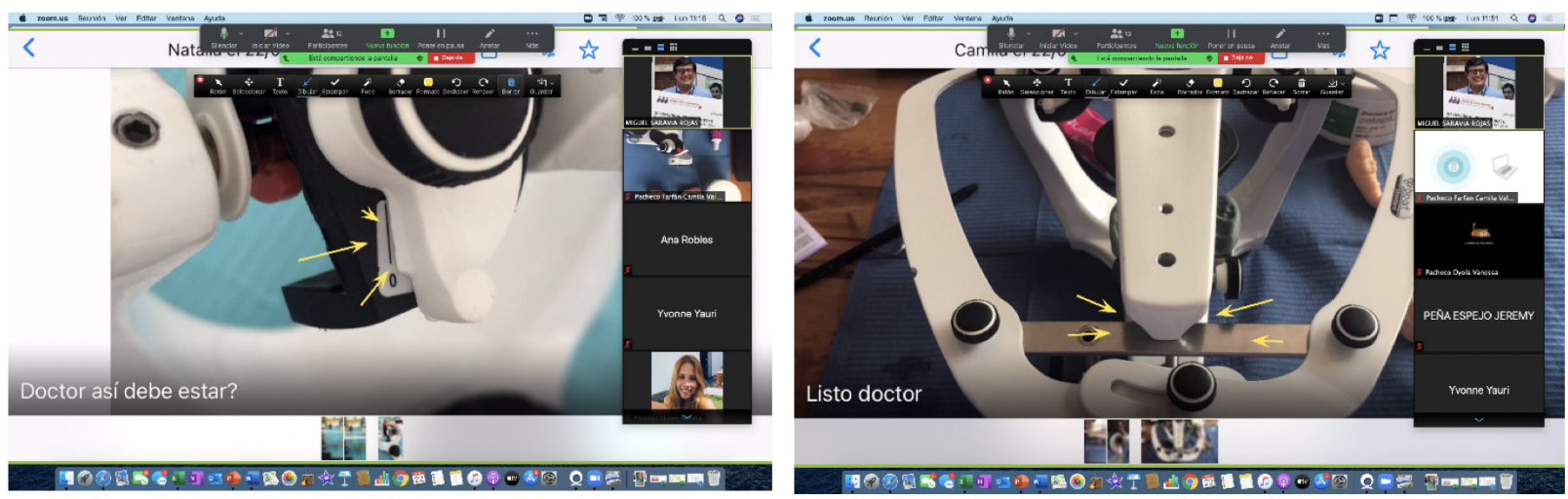

Figura 4. Uso de la herramienta anotar por el estudiante como medio de comunicación con el docente

En relación a las plataformas de vídeo conferencias utilizada alguna vez por los docentes se encontró que el $100 \%$ de los docentes que contestaron la encuesta han utilizado la plataforma zoom, seguido del aplicativo WhatsApp con un $85,7 \%$, el uso de Google meet está en un $42,9 \%$ y otras plataformas como Skype, Facetime y Microsoft team en menor porcentajes (gráfico 1). El comportamiento de los estudiantes fue similar encontrándose que el 100\% ha usado la plataforma Zoom, seguido del aplicativo WhatsApp con un $71,4 \%$, Google meet con un $38,8 \%$ y las otras plataformas en menor porcentajes (gráfico 2).

Tanto los docentes como los estudiantes utilizaron la plataforma de vídeo conferencia para actividades académicas, sociales, laborales y consultas médicas: el $100 \%$ de los docentes y estudiantes lo utilizaron para actividades académicas, en relación al uso en actividades sociales el 71,4\% de docentes señaló que lo utilizaba para este fin, frente al $75,5 \%$ en el caso de estudiantes. En cuanto a las actividades laborales el $64,3 \%$ de los docentes utilizaron la plataforma de vídeo conferencia frente al $18,4 \%$ de los estudiantes y para consultas médicas; como era de esperarse, el 50\% de los docentes manifestó que utilizo la plataforma

Gráfico 1. Plataformas de video conferencia utilizado alguna vez por los docentes.

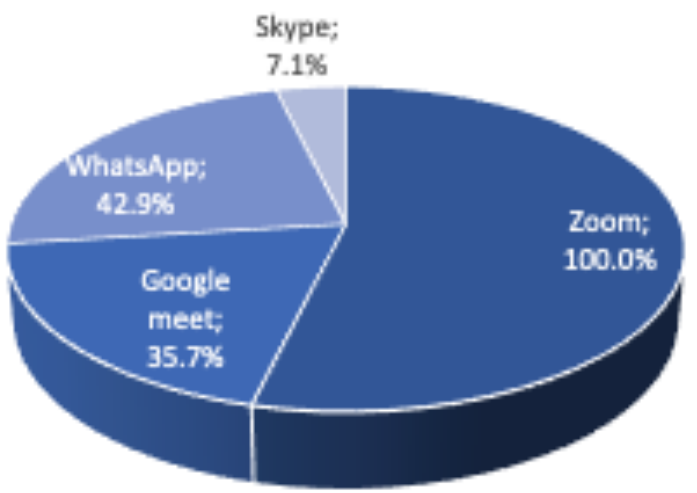


Gráfico 2. Plataformas de vídeo conferencia utilizado alguna vez por los estudiantes.

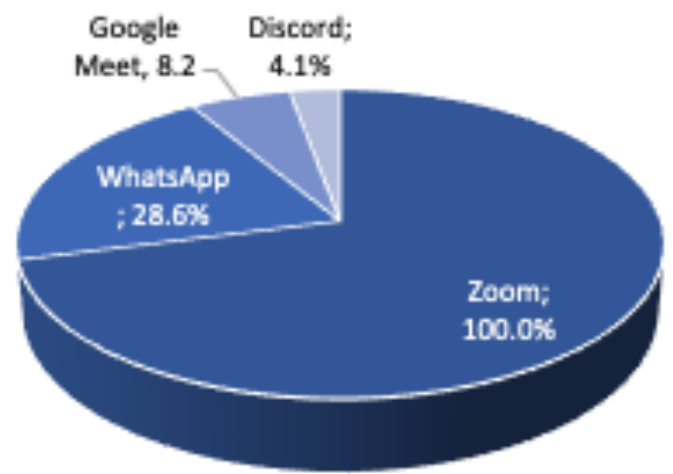

Gráfico 3. Actividades en las que el docente utiliza vídeo conferencia.

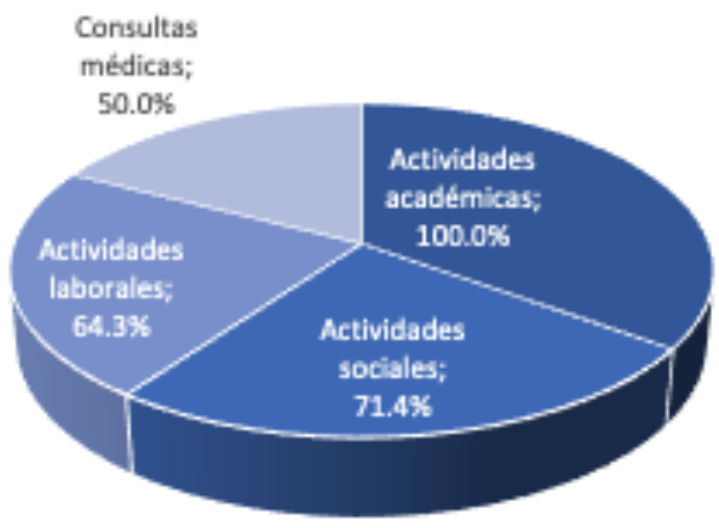

Gráfico 4. Actividades en las que el estudiante utiliza vídeo conferencia.

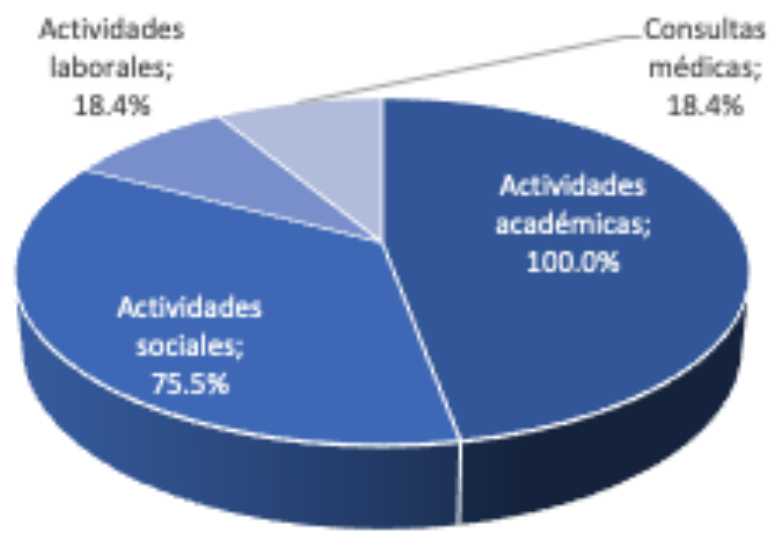

de video conferencia para una consulta médica versus el 18,4\% de los estudiantes (gráfico 3 y 4).

En relación a la herramienta pizarra y anotar el $64 \%$ de los docentes que respondieron la encuesta lo conocían y en el caso de estudiantes el $65 \%$ conocían esta herramienta.

En cuanto a las herramientas más útiles de la plataforma Zoom, el 92,9\% de los docentes consideraron que era el compartir pantalla, seguido por el de formación de grupos en un 78.6\%, el envío de archivos a través del chat con un 50\%, el 42,9\% considera a la herramienta anotar (gráfico 5). En el caso de estudiantes el 93,9\% considera que compartir pantalla era la herramienta más útil del Zoom, seguido también por la formación de grupos en un $85,7 \%$, la herramienta anotar con un 38,8\% y el envío de archivos en un 26,5\% (gráfico 6).

Los dispositivos que más utilizan los docentes para conectarse son: laptop en un 64,3\%, seguido de una computadora personal (PC) con un 21,4\% y el $14,3 \%$ utilizo con mayor frecuencia el celular para conectarse (gráfico 7).

Gráfico 5. Percepción de los docentes sobre la utilidad de las herramientas del zoom.

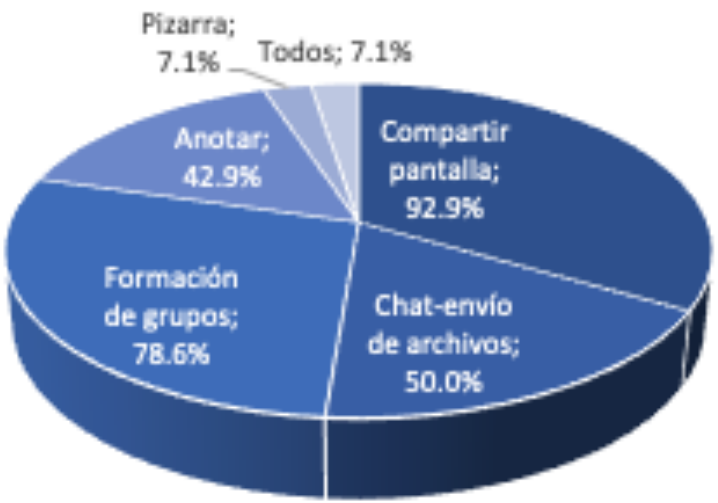

Gráfico 6. Percepción de los estudiantes sobre la utilidad de las herramientas del zoom.

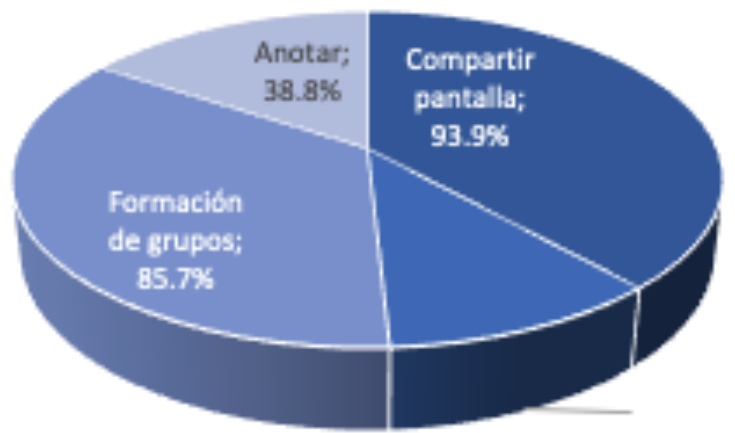


En el caso de los estudiantes el 69,4\% utiliza una laptop, seguido del celular en un $10,2 \%$ y un $8,2 \%$ lo hace desde una PC (gráfico 8).

Adicionalmente se les preguntó sobre las actividades que consideran que debe mantenerse a través de la plataforma Zoom cuando se retorne a la presencialidad y en opinión de los estudiantes el $87,8 \%$ considera que las clases teóricas deben mantenerse a través del Zoom, seguido por las presentaciones de casos clínicos con un $61,2 \%$, el $75,5 \%$ consideró que las tutorías deben seguir realizándose vía Zoom, y el $67,3 \%$ para la consejería psicológica (tabla 1). En este mismo punto el $71,4 \%$ de los docentes que respondieron a la encuesta consideraron que las clases teóricas deben mantenerse utilizando la plataforma Zoom, el $92,5 \%$ para las tutorías, seguido por un $50 \%$ que considera que para todas las presentaciones de caso deben continuar a través de la plataforma Zoom, el $42,9 \%$ para la consejería psicológica (tabla 2).

Gráfico 7. Dispositivo más utilizado por los docentes para conectarse al zoom.

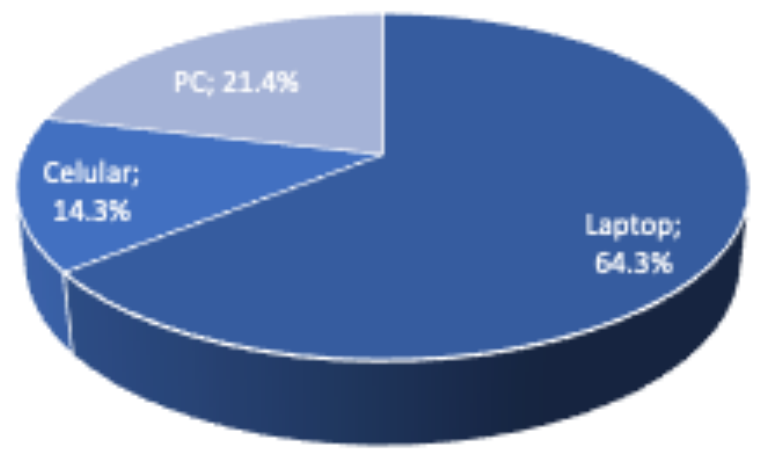

Gráfico 8. Dispositivo más utilizado por los estudiantes para conectarse al zoom

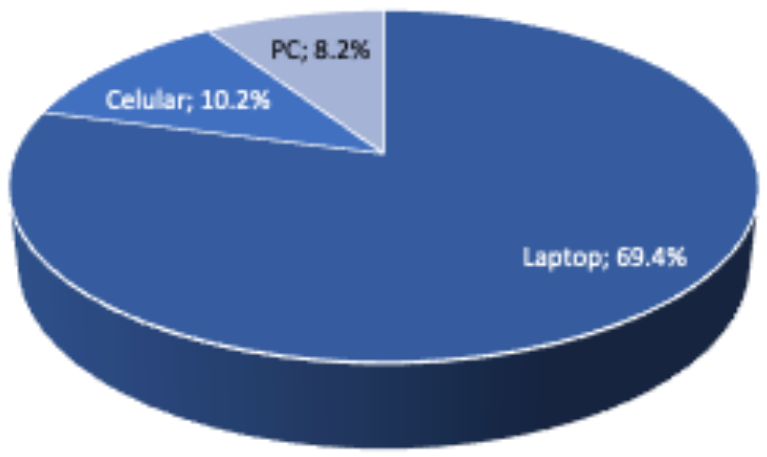

Tabla 1. Actividades que los docentes consideran que debe seguir utilizando el zoom cuando se retorne a la presencialidad

\begin{tabular}{lr}
\hline Actividades & $\mathbf{\%}$ \\
\hline Clases teóricas & 71,4 \\
Todas las presentaciones de casos clínicos & 50,0 \\
Algunas presentaciones de casos clínicos & 42,9 \\
Algunas prácticas en maquetas & 28,6 \\
Todas las prácticas en maquetas & 14,3 \\
Consejería psicológica & 42,9 \\
Tutorías & 92,9 \\
Asesorías & 7,1 \\
\hline
\end{tabular}

Tabla 2. Actividades que los estudiantes consideran que debe seguir utilizando el zoom cuando se retorne a la presencialidad

\begin{tabular}{|l|r|}
\hline Actividades & $\%$ \\
\hline Clases teóricas & 87,8 \\
Todas las presentaciones de casos clínicos & 61,2 \\
Algunas presentaciones de casos clínicos & 20,4 \\
Algunas prácticas en maquetas & 24,5 \\
Todas las prácticas en maquetas & 10,2 \\
Consejería psicológica & 67,3 \\
Tutorías & 75,5 \\
\hline
\end{tabular}

\section{CONCLUSIONES}

La experiencia de usar una plataforma de video conferencia como el Zoom ha sido muy valiosa como instrumento para poder generar aprendizajes significativos en los procedimientos restauradores directos e indirectos. Las herramientas que posee lo hace altamente versátil para poder realizar instrucciones a los alumnos, así como para poder realizar indicaciones precisas y claras en cuanto a los pasos procedimentales que el estudiante debe realizar según la rutina establecida. Una experiencia inédita en simulación con maqueta-UPCH vía remota ha sido realizada con singular éxito. La herramienta pizarra y anotar del Zoom ha demostrado su total utilidad asi como la herramienta de sección de grupos. Tanto docentes como estudiantes usan con mucha frecuencia la laptop como el principal medio de interacción. Para lograr una comunicación efectiva la fotografía y el envío usando aplicativos de imagen y audio fueron muy valiosos para poder cerrar un círculo de 
comunicación efectivo, nos referimos al aplicativo de mensajería instantánea WhatsApp (13).

\section{Correspondencia}

Miguel Ángel Saravia-Rojas

Correo electrónico: miguel.saravia@upch.pe

\section{REFERENCIAS BIBLIOGRÁFICAS}

1. Vera F. Impacto de las plataformas de videoconferencia en la educación superior en tiempos de COVID-19. Revista Electrónica Transformar. 2021; 2(1): 41-57.

2. Ranasinghe L, Wright L. Video lectures versus live lectures: competing or complementary? Med Educ Online. 2019; 24(1): 1574522. doi: 10.1080/10872981.2019.1574522

3. Alfadda HA, Mahdi HS. Measuring Students' Use of Zoom Application in Language Course Based on the Technology Acceptance Model (TAM). J Psycholinguist Res. 2021; 50: 883-900. Doi: 10.1007/s10936-02009752-1

4. Rahayu, D. Students' e-learning experience through a synchronous Zoom web conference system. Journal of ELT Research. 2020; 5(1): 68-79.

5. Dharma HRC, Asmarani D, Dewi UP. Basic Japanese grammar and conversation e-learn- ing through Skype and Zoom online application. Procedia Computer Science. 2017; 116: 267-273. Doi:10.1016/j. procs.2017.10.055.

6. Zoom Video Communications, Inc. Zoom Video Communications. California: Zoom Video Communications, Inc; 2021.

7. McClendon C, Neugebauer RM, King A. Grit, growth mindset, and deliberate practice in online learning. Journal of Instructional Research. 2017; 8: 8-17. Doi: 10.9743/JIR.2017
8. Lowenthal P, Borup J, West R, Archambault L. Thinking beyond Zoom: Using asynchronous video to maintain connection and engagement during the COVID-19 pandemic. Journal of Technology and Teacher Education. 2020; 28(2), 383-391.

9. Ferns S, Cappon A, Duff A, et al. Technology tools for teaching in higher education, the practical handbook series. Canadá: Centre for Higher Education Research, Policy \& Practice; 2020. (Citado el 2 de agosto del 2021) Disponible en https://ecampusontario. pressbooks.pub/techtoolsforteaching/frontmatter/ technology-tools-for-teaching-in-higher-education/

10. Archibald MM, Ambagtsheer RC, Casey MG, Lawless M. Using Zoom Videoconferencing for qualitative data collection: Perceptions an expriences of researchers and participants. International Journal of Qualitative methods. 2019; 18:1-8, DOI: 10.1177/1609406919874596

11. Hernandez JA, Perez R, Vallejo -Trujillo LS. Estudio comparativo de herramientas colaborativas de video conferencias y su impacto en programas de posgrado presenciales: Caso de estudio Universidad Pública Estatal. Memoria del Congreso Internacional de Investigación Academia Journals Tabasco. 2017;9(3):29-31.

12. Islam M Kim D, Kwon M A. comparison of two forms of instruction: pre-recorded video lectures vs live zoom lectures for education in the business management field. Sustainability. 2020;12(19): 1-11 8149, doi:10.3390/su12198149

13. Saravia M, Fukuhara M, Geng-Vivanco, R. Orejuela, F. Student's perception of the impact of the video demostrations shared through WhatsApp in their clinical performance. International Journal of Social Sciences \& Educational Studies. 2021;8(3)30-42. Doi:10.23918/ijsses.v8i3p1

Recibido : 03-08-2021

Aceptado : 30-08-2021 